\title{
Approximation schemes for the study of multi-band Gutzwiller wave functions
}

\author{
Jörg Bünemann ${ }^{1}$, Thorben Linneweber ${ }^{2}$, and Florian Gebhard ${ }^{*}, 1$ \\ ${ }^{1}$ Fachbereich Physik, Philipps Universität, Renthof 6, 35032 Marburg, Germany \\ ${ }^{2}$ Lehrstuhl für Theoretische Physik II, Technische Universität Dortmund, D-44227 Dortmund, Germany
}

Received March 26, 2016, revised XXXX, accepted XXXX

Published online XXXX

Key words: Multi-band Hubbard models, Gutzwiller wave functions, Superconductivity

* Corresponding author: e-mail florian.gebhard@physik.uni-marburg.de

The minimum of the Gutzwiller energy functional depends on the number of parameters considered in the variational state. For a three-orbital Hubbard model we find that the frequently used diagonal Ansatz is very accurate in high-symmetry situations. For lower symmetry, induced by a crystal-field splitting or the spin-orbit coup- ling, the discrepancies in energy between the most general and a diagonal Gutzwiller Ansatz can be quite significant. We discuss approximate schemes that may be employed in multi-band cases where a minimization of the general Gutzwiller energy functional is too demanding numerically.
1 Introduction Transition metals like iron and nickel and their compounds, e.g., the iron pnictides, display a variety of intriguing phenomena such as magnetism and high-temperature superconductivity [1,2]. Since these effects are caused by the strong Coulomb interaction in the narrow $3 d$ bands, it is a common observation that stateof-the-art ab-initio methods do not provide a satisfactory description of these materials' electronic properties [3]. In sufficiently realistic models for transition-metal compounds, one needs to take into account the local Coulomb interaction in all partially filled $3 d$ orbitals. Hence, multiband Hubbard models constitute the minimal models for an adequate description of this class of materials.

Most numerical methods that have been applied successfully for single-band models, e.g., exact diagonalization or the density-matrix renormalization-group method, are not applicable for multi-band models because the Hilbert space dimension depends exponentially on the number of orbital degrees of freedom $n_{\mathrm{o}}$. A numerical technique that permits the study of a single-band model with $L$ lattice sites, can tackle only of the order of $L^{1 / n_{\text {o }}}$ sites in a system with $n_{\mathrm{o}}$ orbitals. Hence, now and in the foreseeable future, the investigation of multi-band models has to rely on appropriate approximations.
A useful method for the investigation of multi-band Hubbard models is based on the Gutzwiller variational approach [4,5]. Gutzwiller wave functions systematically improve Hartree-Fock wave functions by including correlation operators that suppress energetically unfavorable atomic states ('multiplets').

In contrast to Hartree-Fock wave functions, the analytical evaluation of expectation values for Gutzwiller wave functions poses a difficult many-body problem so that additional approximations are mandatory. Most often used in this context is the 'Gutzwiller approximation' that corresponds to an exact evaluation of expectation values in the controlled limit of infinite spatial dimensions [6,7]. The Gutzwiller approximation was applied in many studies of multi-band models, for example on iron pnictides [8,9]. It can be improved systematically by using a diagrammatic technique [7, 10]; this perturbative approach was successfully applied to study Fermi-surface deformations, quasiparticle band structures, and $d$-wave superconductivity in single-band Hubbard models [10,11,12,13], periodic Anderson models [14,15], and t-J [16] and multi-band models [17].

In calculations for multi-band models, a simplified Ansatz is frequently used for the Gutzwiller wave func- 
tion where only the weight of local multiplet states can be varied but not their composition ('diagonal Ansatz'). This variational restriction is imposed because the number of variational parameters is at most $2^{2 n_{\mathrm{o}}}$ for the diagonal Ansatz and at most $\left(2^{2 n_{\mathrm{o}}}\right)^{2}$ for the non-diagonal Ansatz. Since the maximal number of variational parameters that can be handled numerically is of the order of $10^{3}$, the parameter space in a non-diagonal Ansatz would become prohibitively large in models with more than three orbitals per lattice site, $n_{\mathrm{o}}>3$. However, in low-symmetry situations or in the presence of a spin-orbit coupling, a diagonal Ansatz may introduce a significant error for certain ground-state properties, e.g., the magnetic anisotropy. Therefore, it must be improved by taking into account the 'most relevant' non-diagonal variational parameters.

It is the purpose of this work to investigate the limitations of a diagonal Ansatz, and to discuss some strategies to improve it systematically. The ideal model for such a study is a three-orbital model, where a minimization of the most general Gutzwiller energy functional is possible and thus provides a benchmark for all kinds of approximations.

Our work is organized as follows. In Sect. 2 we introduce our three-band Hubbard model and briefly discuss the Gutzwiller variational approach. In Sect. 3 we use a crystal-field splitting and the spin-orbit coupling to illustrate the necessity for non-diagonal variational parameters in the Gutzwiller Ansatz. In Sect. 4 we discuss numerical strategies that could be used in cases where the minimization of the most general energy functional is not possible numerically. A brief summary in Sect. 5 5 closes our presentation.

2 Models and Method In this work we use Gutzwiller wave functions to study the ground-state properties of a three-band Hubbard model. First, we introduce the Hamiltonian, and discuss the variational wave functions next.

2.1 Multi-band Hubbard model We study general multi-band Hubbard models of the form

$$
\hat{H}=\hat{H}_{0}+\sum_{i} \hat{H}_{i ; \mathrm{loc}},
$$

where $\hat{H}_{0}$ denotes the electrons' kinetic energy and $\hat{H}_{i \text {; loc }}$ describes the local Hamiltonian on site $i$ of our simplecubic lattice with $L$ sites.

To be definite, we consider a three-band Hubbard model where electrons move between orbitals $b$ and $b^{\prime}$ on sites $i$ and $j(i \neq j)$. In second quantization the kinetic energy reads

$$
\hat{H}_{0}=\sum_{i \neq j} \sum_{\sigma, \sigma^{\prime}} t_{i, j}^{\sigma, \sigma^{\prime}} \hat{c}_{i, \sigma}^{\dagger} \hat{c}_{j, \sigma^{\prime}}
$$

where we introduced the combined spin-orbital index

$$
\sigma \equiv(b, s), b \in\{1,2,3\}, s \in\{\uparrow, \downarrow\} \text {. }
$$

For our calculations we use the Slater-Koster parameters [18]

$$
\begin{aligned}
t_{\pi}^{(1),(2),(3)} & =0.3,-0.1,0.025 \\
t_{\sigma}^{(2),(3)} & =0.1,0.01 \\
t_{\delta}^{(1),(2),(3)} & =0.1,-0.025,0.02
\end{aligned}
$$

for the electron transfers up to 3rd nearest neighbors.

The local Hamiltonian is given by

$$
\begin{aligned}
\hat{H}_{i ; \mathrm{loc}}= & \sum_{\sigma, \sigma^{\prime}} \epsilon_{\sigma, \sigma^{\prime}} \hat{c}_{i, \sigma}^{\dagger} \hat{c}_{i, \sigma^{\prime}} \\
& +\sum_{\sigma_{1}, \sigma_{2}, \sigma_{3}, \sigma_{4}} U^{\sigma_{1}, \sigma_{2}, \sigma_{3}, \sigma_{4}} \hat{c}_{i, \sigma_{1}}^{\dagger} \hat{c}_{i, \sigma_{2}}^{\dagger} \hat{c}_{i, \sigma_{3}} \hat{c}_{i, \sigma_{4}}
\end{aligned}
$$

The single-particle energies encoded in the Hermitian matrix $\tilde{\epsilon}$ describe the crystal fields and the local spin-orbit coupling. For three (degenerate) $t_{2 \mathrm{~g}}$ orbitals, the two-particle Coulomb interaction in (5) has the form

$$
\begin{aligned}
2 \hat{H}_{i ; \mathrm{C}}= & U \sum_{b, s} \hat{n}_{i, b, s} \hat{n}_{i, b, \bar{s}} \\
& +\sum_{\substack{b(\neq) b^{\prime} \\
s, s^{\prime}}}\left(U-2 J-\delta_{s, s^{\prime}} J\right) \hat{n}_{i, b, s} \hat{n}_{i, b^{\prime}, s^{\prime}} \\
& +J \sum_{b(\neq) b^{\prime}}\left[\left(\hat{c}_{i, b, \uparrow}^{\dagger} \hat{c}_{i, b, \downarrow}^{\dagger} \hat{c}_{i, b^{\prime}, \downarrow} \hat{c}_{i, b^{\prime}, \uparrow}+\text { h.c. }\right)\right. \\
& \left.\quad+\sum_{s} \hat{c}_{i, b, s}^{\dagger} \hat{c}_{i, b^{\prime}, \bar{s}}^{\dagger} \hat{c}_{i, b, \bar{s}} \hat{c}_{i, b^{\prime}, s}\right],
\end{aligned}
$$

where we use the convention $\uparrow=\downarrow, \bar{\downarrow}=\uparrow$, and $\hat{n}_{i, b, s}=$ $\hat{c}_{i, b, s}^{\dagger} \hat{c}_{i, b, s}$ counts the electrons with spin $s$ in orbital $b$ on site $i$. We can diagonalize the local Hamiltonian (5) at least numerically,

$$
\hat{H}_{i ; \mathrm{loc}}=\sum_{\Gamma} E_{\Gamma} \hat{m}_{i, \Gamma} \quad, \quad \hat{m}_{i, \Gamma} \equiv|\Gamma\rangle_{i i}\langle\Gamma|
$$

and determine its eigenstates ('multiplet states') $|\Gamma\rangle_{i}$.

2.2 Gutzwiller wave functions The general multiband Gutzwiller wave function has the form

$$
\left|\Psi_{\mathrm{G}}\right\rangle=\prod_{i} \hat{P}_{i}\left|\Psi_{0}\right\rangle
$$

where $\left|\Psi_{0}\right\rangle$ is a normalized single-particle product state, i.e., a Hartree-Fock wave function. The simplest, and most frequently used, form of the local Gutzwiller correlator is the 'diagonal Ansatz',

$$
\hat{P}_{i}=\sum_{\Gamma} \lambda_{\Gamma} \hat{m}_{i, \Gamma} .
$$

This wave function contains real variational parameters $\lambda_{\Gamma}$ that allow us to optimize the probability

$$
m_{i, \Gamma}^{\mathrm{G}} \equiv\left\langle\hat{m}_{i, \Gamma}\right\rangle_{\Psi_{\mathrm{G}}}
$$


for the state $|\Gamma\rangle_{i}$ to be occupied in the variational ground state (8). Here, $\langle\ldots\rangle_{\Psi_{\mathrm{G}}}$ denotes expectation values with respect to $\left|\Psi_{\mathrm{G}}\right\rangle$.

Two problems arise from the 'diagonal' Ansatz (9). First, the spectrum of $\hat{H}_{i \text {;oc }}$ is usually degenerate and its eigenstates are therefore not uniquely defined. Second, and more importantly, the diagonal operator (9) is not the most general Ansatz. Therefore, it is not clear to what extent physical results change when we work with the general local correlation operator

$$
\hat{P}_{i}=\sum_{\Gamma, \Gamma^{\prime}} \lambda_{\Gamma, \Gamma^{\prime}}|\Gamma\rangle_{i i}\left\langle\Gamma^{\prime}\left|\equiv \sum_{\Gamma_{d}} \lambda_{\Gamma_{d}}\right| \Gamma_{d}\right\rangle_{i i}\left\langle\Gamma_{d}\right|,
$$

which contains a (Hermitian) matrix $\tilde{\lambda}$ of (complex) variational parameters. This Ansatz allows us to optimize the occupation and also the composition of the eigenstates $\left|\Gamma_{d}\right\rangle_{i}$ of $\hat{P}_{i}$

The task is the calculation of the variational groundstate energy functional

$$
E_{\mathrm{G}}\left(\tilde{\lambda},\left|\Psi_{0}\right\rangle\right)=\langle\hat{H}\rangle_{\Psi_{\mathrm{G}}}
$$

and its minimization with respect to the variational parameters. For later use, we also define the kinetic energy and the local energy per lattice site as the expectation values of $\hat{H}_{0} / L$ and of $\hat{H}_{i ; \text { loc }}$ at the optimal variational parameters,

$$
E_{\mathrm{kin}}=\left\langle\hat{H}_{0} / L\right\rangle_{\Psi_{\mathrm{G}, \mathrm{opt}}} \quad, \quad E_{\mathrm{loc}}=\left\langle\hat{H}_{i ; \mathrm{loc}}\right\rangle_{\Psi_{\mathrm{G}, \mathrm{opt}}} .
$$

2.3 Gutzwiller approximation It is most convenient for the evaluation of Gutzwiller wave functions in infinite dimensions to impose the following (local) constraints [19. 20],

$$
\begin{aligned}
\left\langle\hat{P}_{i}^{\dagger} \hat{P}_{i}\right\rangle_{\Psi_{0}}-1 & \equiv g_{1}^{\mathrm{c}}\left(\tilde{\lambda},\left|\Psi_{0}\right\rangle\right)=0, \\
\left\langle\hat{c}_{\sigma}^{\dagger} \hat{P}_{i}^{\dagger} \hat{P}_{i} \hat{c}_{\sigma^{\prime}}\right\rangle_{\Psi_{0}}-C_{\sigma^{\prime}, \sigma} & \equiv g_{\sigma, \sigma^{\prime}}^{\mathrm{c}}\left(\tilde{\lambda},\left|\Psi_{0}\right\rangle\right)=0 .
\end{aligned}
$$

Here, we introduced the local density matrix $\tilde{C}_{i}$ with the elements

$$
C_{i ; \sigma, \sigma^{\prime}}=\left\langle\hat{c}_{i, \sigma^{\prime}}^{\dagger} \hat{c}_{i, \sigma}\right\rangle_{\Psi_{0}} .
$$

The constraints and other expectation values with the single-particle product state $\left|\Psi_{0}\right\rangle$ can be evaluated by means of Wick's theorem.

As shown in Refs. [19,20], it is possible to derive analytical expressions for the ground-state energy functional $E_{\mathrm{G}}$ in eq. (12) in the limit of infinite spatial dimensions. An application of this energy functional to finite-dimensional systems is usually termed 'Gutzwiller approximation'. The ground-state energy is a functional of the variational-parameter matrix $\tilde{\lambda}$ and of the single-particle wave function $\left|\Psi_{0}\right\rangle$. It can be shown that the optimal state $\left|\Psi_{0}\right\rangle$ is the ground-state of an effective single-particle Hamiltonian,

$$
\hat{H}_{0}^{\mathrm{eff}}=\sum_{i, j} \sum_{\sigma, \sigma^{\prime}}\left(\bar{t}_{i, j}^{\sigma, \sigma^{\prime}}+\delta_{i, j} \eta_{\sigma, \sigma^{\prime}}\right) \hat{c}_{i, \sigma}^{\dagger} \hat{c}_{j, \sigma^{\prime}},
$$

where $\bar{t}_{i, j}^{\sigma, \sigma^{\prime}}$ are renormalized electron transfer parameters and the matrix $\tilde{\eta}$ contains effective crystal fields and spinorbit couplings [21]. Explicit expressions for our threeband model can be found in Ref. [22]. To study solely the importance of the non-diagonal elements in the variationalparameter matrix $\tilde{\lambda}$, we fix $\left|\Psi_{0}\right\rangle$ in most of the following numerical calculations and do not optimize it. If not specified explicitly otherwise, the state $\left|\Psi_{0}\right\rangle$ is chosen as the ground state of (17) with $\bar{t}_{i, j}^{\sigma, \sigma^{\prime}}=t_{i, j}^{\sigma, \sigma^{\prime}}$ and $\eta_{\sigma, \sigma^{\prime}}=\epsilon_{\sigma, \sigma^{\prime}}$.

In our numerical optimization, we do not fulfill the constraints exactly but (eventually) with high numerical accuracy, see Ref. [22] where we describe the minimization algorithm in detail. To measure the deviation from the exact constraints, we define

$$
|\Delta g|^{2}=\left|g_{1}^{\mathrm{c}}\right|^{2}+\sum_{\sigma, \sigma^{\prime}}\left|g_{\sigma, \sigma^{\prime}}\right|^{2}
$$

At the minimum of the energy functional, we verify that the $\Delta g<10^{-9}$.

3 Results For the symmetric model with $\epsilon_{\sigma, \sigma^{\prime}}=0$ in (5), the multiplet states are either degenerate or they belong to different representations of the atomic pointsymmetry group. Hence, in this case, the inclusion of non-diagonal elements in the variational-parameter matrix does not lead to any energy gain. Only when we reduce the symmetry, the importance of non-diagonal elements in the variational-parameter matrix $\tilde{\lambda}$ can be studied. In this section, we consider two forms of symmetry reductions, a crystal field splitting (CFS) and the spin-orbit coupling (SOC).

3.1 Crystal field splitting (CFS) We start with a situation where we break the orbital symmetry with a field of the form $\epsilon_{\sigma, \sigma^{\prime}}=\delta_{\sigma, \sigma^{\prime}} \Delta\left(\delta_{b, 1}-\delta_{b, 3}\right)$ for the three orbitals $(\Delta>0)$. In this case we have $\tilde{\eta}=\tilde{\epsilon}$ in eq. 177 from which we determine the single-particle state $\left|\Psi_{0}\right\rangle$. The multiplet states $|\Gamma\rangle$ follow from the diagonalization of $\hat{H}_{i \text {;loc }}$ in eq. (5). We introduce the energies $E^{\text {diag,full for the two }}$ cases of a minimization that includes all ('full') or just diagonal ('diag') elements of the variational-parameter ma$\operatorname{trix} \tilde{\lambda}$.

In Fig. 1 we display $\Delta E_{\text {kin }} \equiv-\left(E_{\mathrm{kin}}^{\mathrm{diag}}-E_{\mathrm{kin}}^{\mathrm{full}}\right)$ and $\Delta E_{\mathrm{loc}} \equiv E_{\text {loc }}^{\text {diag }}-E_{\text {loc }}^{\text {full }}$ for three different values of $\Delta$. Although the differences in these energies are clearly discernible, they are actually relatively small because $E_{\text {kin }}$ is of the order of unity. This holds in particular for the differences in the total energy $\Delta E$ (not shown). Since we reversed the sign in the kinetic energy curve, $\Delta E$ is given as the (small) difference between solid and dashed lines in Fig. (1), $\Delta E=\Delta E_{\text {loc }}-\Delta E_{\text {kin }}$; the energy gain by using the full set of variational parameters is seen to be of the order of $\mathcal{O}\left(10^{-3}\right)$, an order of magnitude smaller than the differences in kinetic and local energies.

The success of the diagonal form of the Gutzwiller correlator hinges on the proper choice of the basis states $|\Gamma\rangle$. 


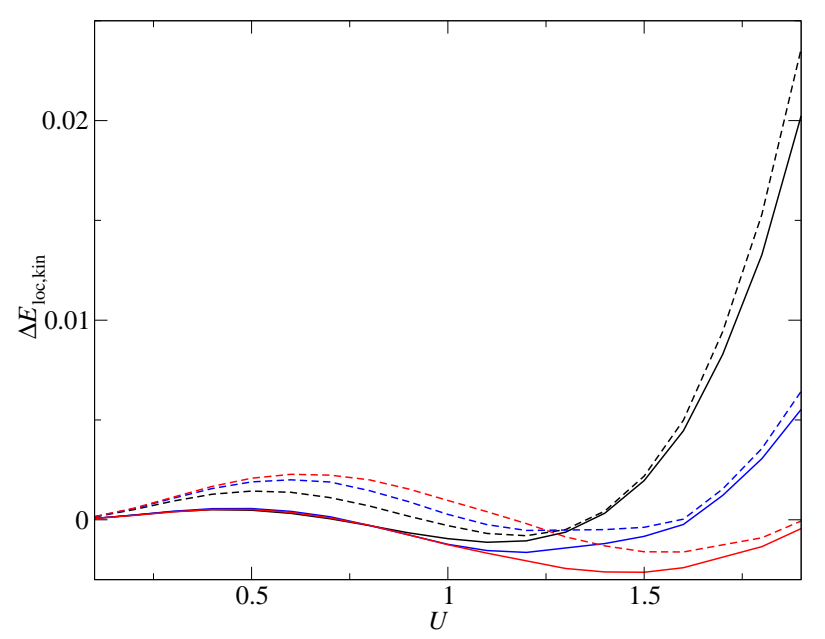

Figure 1 Energy differences $\Delta E_{\text {kin }}$ (solid lines) and $\Delta E_{\text {loc }}$ (dashed lines) as a function of $U$ with $J / U=0.2$ at half band-filling for CFS $\Delta=0.1$ (black), $\Delta=0.2$ (blue), and $\Delta=0.3$ (red).

They are formed with a crystal-field in $\epsilon_{\sigma, \sigma^{\prime}}$ for the generic $t_{2 \mathrm{~g}}$ orbitals $\phi_{x y} \sim x y, \phi_{x z} \sim x z$, and $\phi_{y z} \sim y z$ for $b=1,2,3$. The results for the energies are less good when we work with basis states $|\Gamma\rangle$ that are less appropriate. To illustrate this point, we introduce a rotated crystal field so that the single-electron orbitals are given by

$$
\begin{aligned}
\bar{\phi}_{1} & =\frac{1}{2}\left(\mathrm{i} \phi_{x y}+(1+\mathrm{i}) \phi_{x z}+\phi_{y z}\right), \\
\bar{\phi}_{2} & =\frac{1}{2}\left(\mathrm{i} \phi_{x y}-(1+\mathrm{i}) \phi_{x z}+\phi_{y z}\right), \\
\bar{\phi}_{3} & =\sqrt{\frac{1}{2}}\left(\mathrm{i} \phi_{x y}-\phi_{y z}\right),
\end{aligned}
$$

and the new multiplet states $|\Gamma\rangle$ are formed using these single-particle orbitals orbitals. Note that we keep $\tilde{\eta}$ diagonal in the original $t_{2 \mathrm{~g}}$-basis in the calculation of $\left|\Psi_{0}\right\rangle$ via eq. 177 so that the single-particle band states and the local single-particle orbitals are not aligned anymore. Consequently, as seen from Fig. 2 the differences in the kinetic and local energies from the diagonal and the full variational Ansatz are larger than in the aligned case, by an order of magnitude. Correspondingly, the total variational energy decreases by several percent when we use the full variational-parameter matrix.

As a first step to improve the variational energies we can introduce non-diagonal variational parameters in the single-particle subspace of the atomic Hamiltonian where $|\Gamma\rangle$ contains only a single electron. The dashed lines in Fig. 2 show the corresponding results for this minimization. As the total energy must improve, the dashed red curve is always below the solid red line. However, the improvement using these additional non-diagonal parameters is marginal. Nevertheless, the inclusion of these terms has

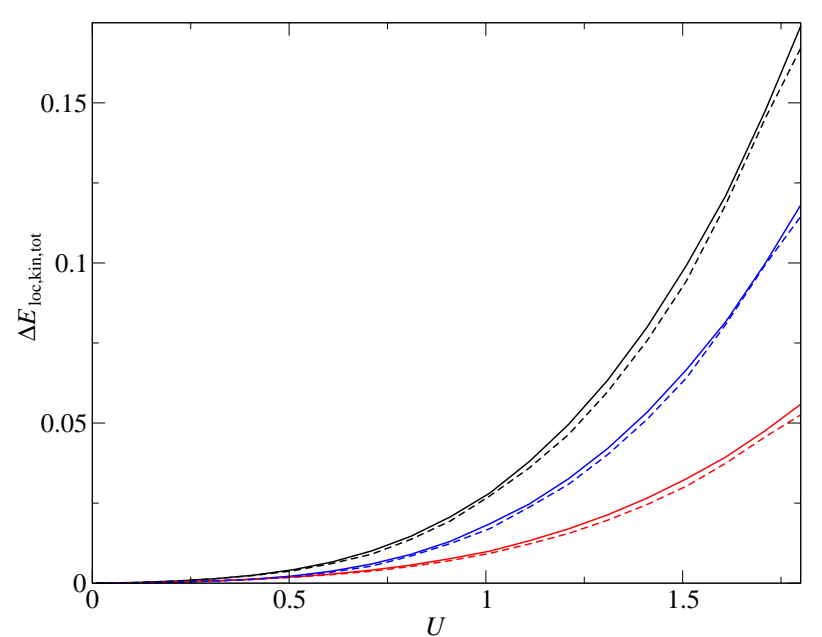

Figure 2 Energy differences $\Delta E_{\text {loc }}$ (black), $\Delta E_{\text {kin }}$ (blue), and $\Delta E_{\text {tot }}$ (red) as a function of $U$ at half band-filling with $J / U=0.2, \Delta=0.2$ (CFS); solid lines: diagonal variational-parameter matrix; dashed lines: non-diagonal variational-parameter matrix in the single-particle subspace (see text).

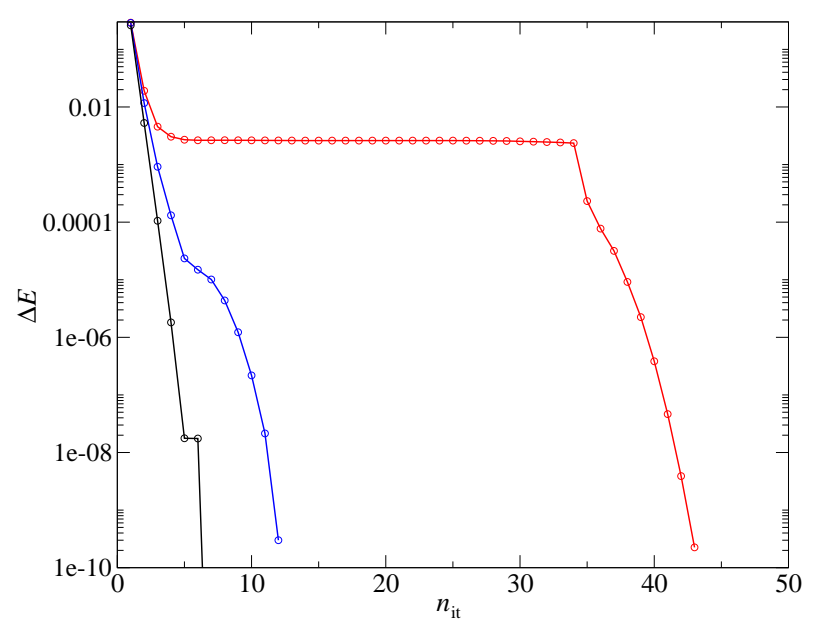

Figure 3 Energy expectation value, relative to the respective ground-state value, at each step of the minimization algorithm for a variational-parameter matrix which allows for all non-diagonal elements (black), non-diagonal elements in the single-particle sector (blue), and only diagonal elements (red) at half band-filling for $U=2, J / U=0.2$, $\Delta=0.2$ (CFS); note the logarithmic scale of the energy axis.

a very positive effect on the convergence of our minimization algorithm, as we shall explain now.

Figure 3 shows, on a logarithmic scale, the convergence of the energy expectation value towards its ground-state value at each step of the minimization algorithm for all three sets of variational-parameter matrices. Apparently, 


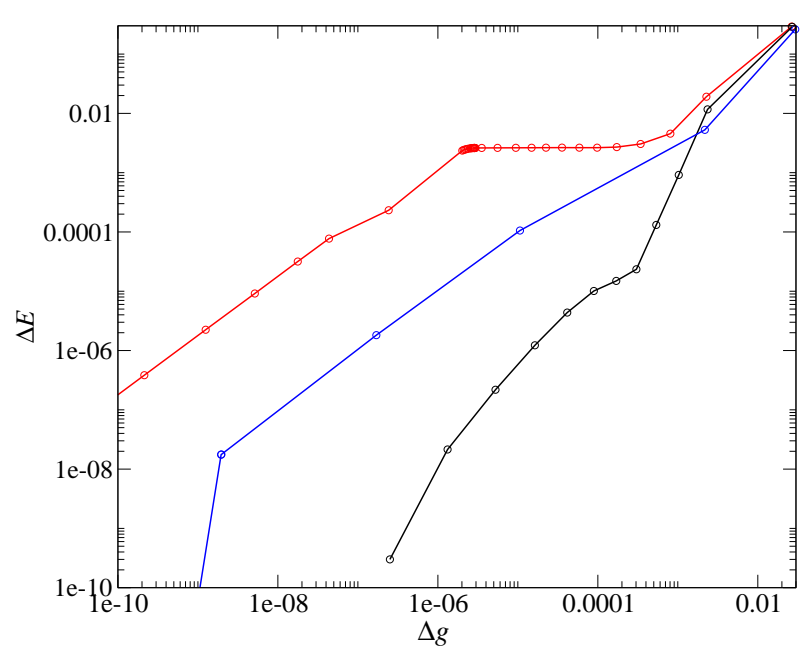

Figure 4 Energy expectation value, relative to the respective ground state value, as a function of the constraint mismatch $\Delta g$ from eq. (18) at each step of the minimization algorithm for a variational-parameter matrix which allows for all non-diagonal elements (black), non-diagonal elements in the single-particle sector (blue), and only diagonal elements (red) at half band-filling for $U=2, J / U=0.2$, $\Delta=0.2$ (CFS); note the logarithmic scale of both axes.

the convergence with a diagonal variational-parameter matrix is very slow when compared to the other two calculations. The main problem with our diagonal matrix is the satisfaction of the constraints (14) and (15). In our 'Penalty and Augmented Lagrangian Method' (PALM) [22, 23] we have to reach quite large values of the penalty parameter $\mu$ before the constraints fall below a certain threshold. This can be seen in Fig. 4 where we show the same energies as in Fig. 3, now as a function of the constraint mismatch parameter $\Delta g$ from eq. (18).

Our investigation of the crystal-field splitting shows that in low-symmetry situations we face two problems when we try not to take into account all elements of the Gutzwiller variational-parameter matrix.

(i) The convergence of the minimization algorithm becomes worse, i.e., it takes more iteration steps to reach convergence.

(ii) The expectation value for the variational ground-state energy noticeably increases. In the worst case, this indicates that the variational state is not flexible enough to describe quantitatively (or even qualitatively) the physics of the underlying Hamiltonian.

Our somewhat artificial example to reduce the local symmetry suggests two possible strategies to solve these problems.

(i) To speed up the convergence, take into account a sufficient number of non-diagonal variational parameters in order to satisfy the constraints.

(ii) Choose a proper basis set of multiplet states $|\Gamma\rangle$.

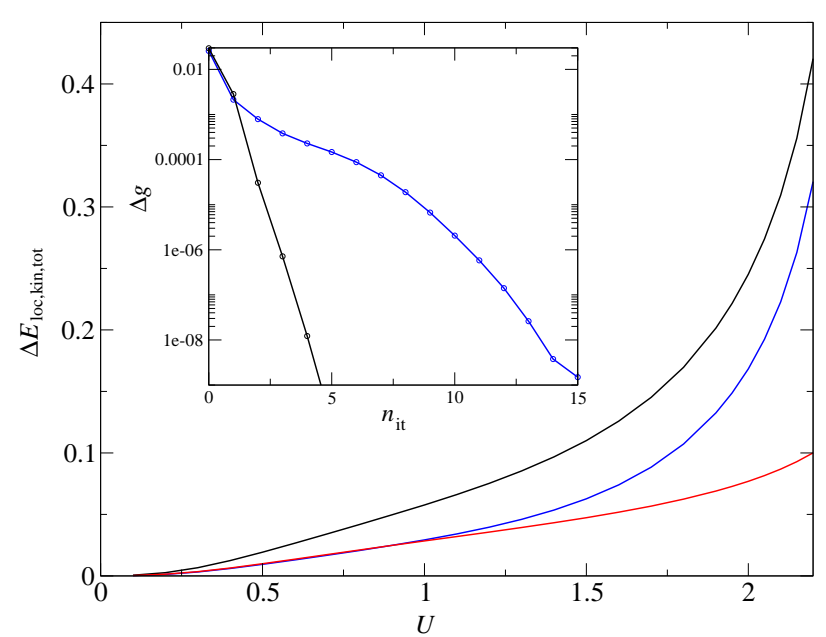

Figure 5 Energy differences $\Delta E_{\text {loc }}$ (black), $\Delta E_{\text {kin }}$ (blue), and $\Delta E_{\text {tot }}$ (red) at half band-filling as a function of $U$ for $J / U=0.2, \zeta=0.2$ (SOC); inset: constraint mismatch as a function of the iteration step $n_{\mathrm{it}}$ of the algorithm with a full (black) and a diagonal (blue) variational-parameter matrix for $U=2$.

In the following section we shall test our strategies against the case where we reduce the symmetry by including the spin-orbit coupling in our local Hamiltonian.

3.2 Spin-orbit coupling (SOC) The spin-orbit coupling provides a physical mechanism that lowers the symmetry of our model with degenerate $t_{2 \mathrm{~g}}$ orbitals. Since it influences the local orbitals, it is an important perturbation and requires a large flexibility in the Gutzwiller wave function. At the same time, it generates small quantitative corrections in the ground-state energy so that the Gutzwiller wave function must be evaluated with high numerical accuracy. The physics of such a system is discussed in detail in Ref. [22]. We will therefore concentrate in the following on the technical problem of convergence and accuracy of our energy minimization.

In the presence of the spin-orbit coupling, the six local spin-orbital states split into two-fold $(j=1 / 2)$ and fourfold $(j=3 / 2)$ degenerate sub-spaces. Similar splittings occur in the multiplet states $|\Gamma\rangle$. Since most of these states remain degenerate, we have to make a certain choice of our basis $|\Gamma\rangle$ when we work with a diagonal variationalparameter matrix. It turns out, however, that in the present calculations this choice is not significant, i.e., we obtain hardly any energy gain by optimizing the energy with respect to the basis $|\Gamma\rangle$.

In any case, the discrepancies in energy can be profound, of the order of ten percent for $U=2$, when we compare results for the full and a diagonal variationalparameter matrix. This can be seen in Fig. 5 where we show the energy differences defined in the previous section for a bare spin-orbit coupling of $\zeta=0.2$ [22]. As in our crystal-field calculations in Sect. 3.1 this inaccuracy 
goes hand in hand with a significantly slower convergence of the minimization algorithm, see the inset of Fig. 5.

As seen from the figure, the discrepancies in the kinetic and potential energies are much worse than those for the total energy. For the kinetic energy, this is reflected by rather different values for the band-width renormalization factors. For example, at $U=2$, the renormalizations are $\left(q_{1 / 2}^{2} \approx 0.72, q_{3 / 2}^{2} \approx 0.64\right)$ for a diagonal variationalparameter matrix and $\left(q_{1 / 2}^{2} \approx 0.62, q_{3 / 2}^{2} \approx 0.61\right)$ for the full variational-parameter matrix. These values do not change much when we carry out a full minimization also with respect to $\left|\Psi_{0}\right\rangle$. The corresponding numbers are $\left(q_{1 / 2}^{2} \approx 0.73, q_{3 / 2}^{2} \approx 0.64\right)$ and $\left(q_{1 / 2}^{2} \approx 0.62\right.$, $\left.q_{3 / 2}^{2} \approx 0.60\right)$, respectively. Therefore, the large differences in the band-width renormalization factors are not compensated by optimizing the corresponding single-particle states $\left|\Psi_{0}\right\rangle$. The results are generic for a full or only partial treatment of the variational-parameter matrix.

Moreover, in both cases the effective spin-orbit coupling in $\hat{H}_{0}^{\text {eff }}[22]$ is quite different, $\bar{\zeta}^{\text {diag }} \approx 0.35$ and $\bar{\zeta}^{\text {full }} \approx 0.22$. As a consequence, the quasi-particle bandstructures that result from the two calculations [24] differ considerably, with respect to their band-widths and their spin-orbit splittings at various high-symmetry points in the Brillouin zone, see Fig. 6

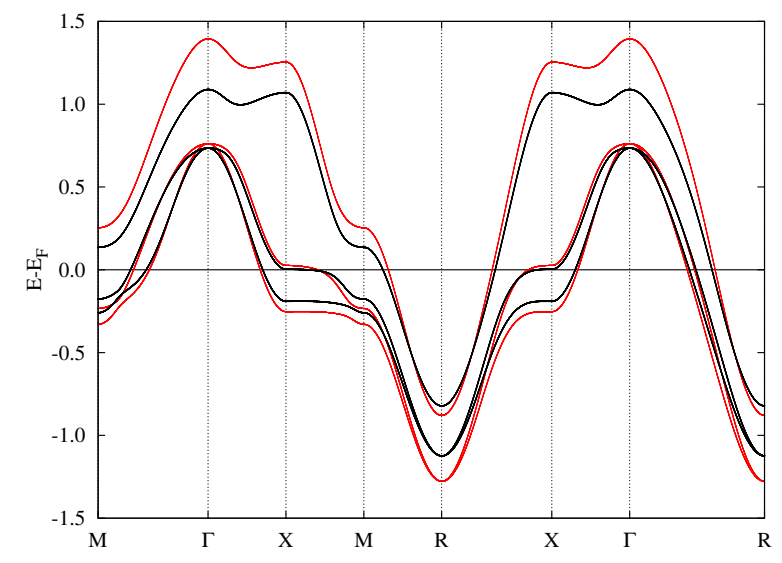

Figure 6 Quasi-particle band structure for our three-band model with spin-orbit coupling along high-symmetry lines in the Brillouin zone at half band-filling for $U=2, \zeta=0.2$ (SOC); red lines: diagonal variational-parameter matrix; black lines: full variational-parameter matrix.

The example of the spin-orbit coupling corroborates our findings in Sect. 3.1 that the variational state with a diagonal variational-parameter matrix is not flexible enough to describe the physics of the underlying Hamiltonian.

4 Approximation strategies As seen in the previous section, the results for expectations values within the Gutz- willer variational method can be rather different for the full and a diagonal variational-parameter matrix. In systems with more than three orbitals, however, it is not possible to take into account all non-diagonal parameters in the numerical minimization. Hence, we have to develop strategies to cope with such situations.

One possible solution is the exploitation of symmetries among the variational parameters, as has been done, e.g., in Refs. [25,26]. Such a solution, however, will only work in systems with a sufficiently large point-group symmetry. As we have shown in Sect. 3, the problem with non-diagonal variational parameters is most acute when the symmetry is low. Hence, a symmetry analysis will only be of limited use.

4.1 Brute-force methods A natural numerical approach to the problem is the inclusion of only a subset of non-diagonal variational parameters $\lambda_{\Gamma, \Gamma^{\prime}}$. Then, it arises the question how to select the parameters that are taken into account. We have tested two different conditions (CON1 and CON2), where we include all parameters for which

$$
\text { CON1: }\left|m_{\Gamma, \Gamma^{\prime}}^{0}\right|>m_{\mathrm{c}}
$$

or

$$
\text { CON2: } m_{\Gamma, \Gamma}^{0} m_{\Gamma^{\prime}, \Gamma^{\prime}}^{0}>m_{\mathrm{c}}^{2} .
$$

Here, we introduced the local expectation value

$$
m_{\Gamma, \Gamma^{\prime}}^{0}=\langle\mid \Gamma\rangle_{i i}\left\langle\Gamma^{\prime} \mid\right\rangle_{\Psi_{0}} .
$$

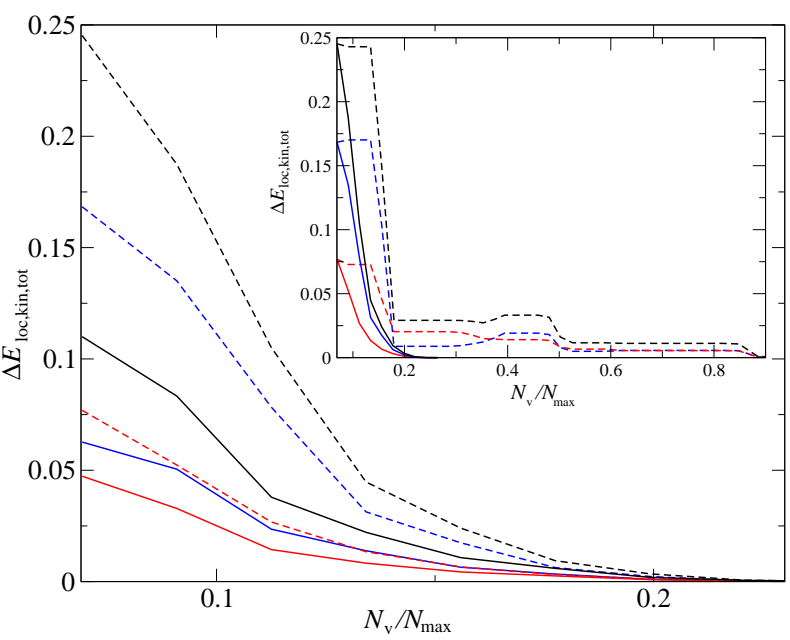

Figure 7 Energy differences $\Delta E_{\text {loc }}$ (black), $\Delta E_{\text {kin }}$ (blue), and $\Delta E_{\text {tot }}$ (red) as a function of $N_{\mathrm{v}}$ (included number of variational parameters) for the cutoff condition CON1 at half band-filling and $J / U=0.2, \zeta=0.2$ (SOC) for $U=$ 1.5 (solid lines) and $U=2$ (dashed lines); inset: same results as in the main figure for $U=2, \mathrm{CON} 1$ (solid lines) and CON2 (dashed lines).

Figure 7 shows the errors in kinetic, local and total energy for $U=1.5,2, J / U=0.2$, and $\zeta=0.1,0.2$ as 
a function of the ratio $N_{\mathrm{v}} / N_{\max }$ of included variational parameters and their maximum number $N_{\max }=924$. Clearly, the method CON1 converges quite rapidly as a function of $N_{\mathrm{v}} / N_{\max }$. Already $20 \%$ of the parameters are sufficient to get a very good agreement with the full calculation. The convergence of CON2 is much worse as can be seen in the inset of Fig. 7 .

The much better convergence of CON1 does not come as a surprise. The point symmetry of the system is still relatively high so that many variational parameters $\lambda_{\Gamma, \Gamma^{\prime}}$ do not enter the energy functional at all. Such parameters are identified automatically and excluded by CON1 because for them we have $m_{\Gamma, \Gamma^{\prime}}^{0}=0<m_{\mathrm{c}}$. In fact, when we lower the symmetry further by introducing an additional crystal field as in Sect. 3.1, the performance of CON1 is less impressive. This can be seen from Fig. 8 where we show the errors in energies for $U=2, J / U=0.2, \zeta=0.2$, and a crystal-field splitting $\Delta=0.2$.

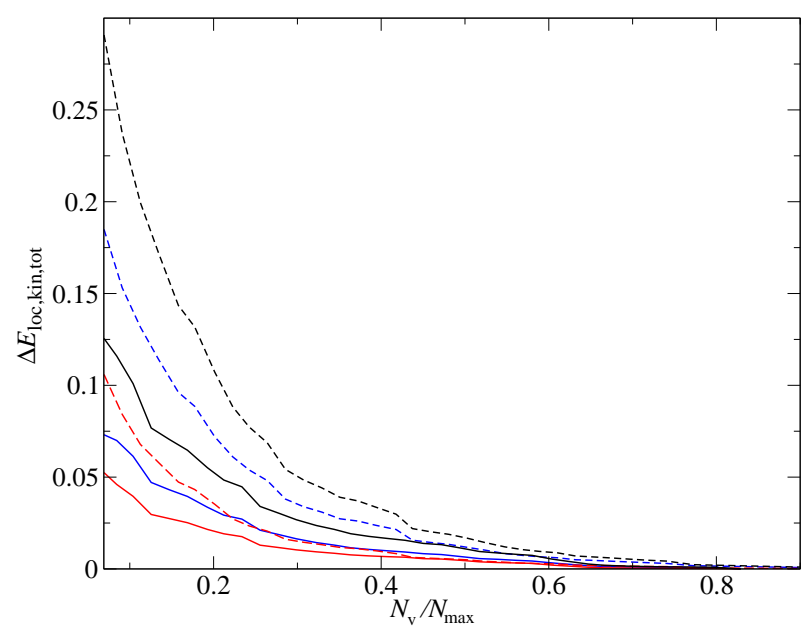

Figure 8 Energy differences $\Delta E_{\text {loc }}$ (black), $\Delta E_{\text {kin }}$ (blue), and $\Delta E_{\text {tot }}$ (red) as a function of $N_{\mathrm{v}}$ (included number of variational parameters) for the cutoff condition CON1 at half band-filling and $J / U=0.2, \zeta=0.2$ (SOC), $\Delta=0.2$ (CFS) for $U=1.5$ (solid lines) and $U=2$ (dashed lines).

4.2 Optimization of the multiplet basis As seen from Fig. 8, the brute-force inclusion of a maximal number of non-diagonal variational parameters may still lead to significant errors in low-symmetry systems with orbital number $n_{\mathrm{O}}>3$. Moreover, unlike in our three-band model, there is no way to estimate systematically the error that is caused by the neglect of some non-diagonal parameters. In the following we develop a more sophisticated algorithm which addresses both of these problems.

If we knew the eigenstates $\left|\Gamma_{d}\right\rangle_{i}$ of the optimum Gutzwiller correlation operator in 111 we would obtain the minimal Gutzwiller ground-state energy by working with a correlation operator that depends only on (in our case 64) diagonal variational parameters. Therefore, it is highly desirable to have an algorithm which systematically improves our multiplet basis $|\Gamma\rangle$ towards $\left|\Gamma_{d}\right\rangle$ in order to make nondiagonal variational parameters increasingly dispensable. To achieve this, we perform the following steps.

(i) Start with some initial local multiplet basis $\left|\Gamma_{0}\right\rangle$.

(ii) Minimize the energy with respect to a variationalparameter matrix that contains a subset of non-diagonal parameters.

(iii) Determine the eigenstates $\left|\Gamma_{1}\right\rangle$ of the optimum variational-parameter matrix $\tilde{\lambda}$ obtained in step (ii).

(iv) Set $\left|\Gamma_{0}\right\rangle=\left|\Gamma_{1}\right\rangle$ and go back to step (ii) until the variational ground-state energy does not improve significantly anymore.

As an illustration, we apply this algorithm to the system with spin-orbit coupling and crystal-field splitting that we analyzed in Fig. 8 As subsets in step (ii), we choose variational parameters that belong to the subspaces with particle numbers $n_{\text {active }}=3, n_{\text {active }}=2,4$, or $n_{\text {active }}=1,5$.

\begin{tabular}{r|c|c|c}
$n_{\text {active }}$ & $\Delta E_{\mathrm{tot}}$ & $t_{\mathrm{CPU}} / t_{\mathrm{CPU}}^{\mathrm{full}}$ & $t_{\mathrm{CPU}}^{\mathrm{CON} 1} / t_{\mathrm{CPU}}^{\text {full }}$ \\
\hline 3 & 0.01771 & 0.028 & 0.10443 \\
$2+4$ & 0.00668 & 0.060 & 0.231013 \\
3 & 0.00412 & 0.171 & 0.300633 \\
$2+4$ & 0.00281 & 0.215 & 0.436709 \\
3 & 0.00204 & 0.266 & 0.322785 \\
$2+4$ & 0.00158 & 0.323 & 0.553797 \\
$1+5$ & 0.00105 & 0.326 & 0.509494 \\
$2+4$ & 0.00103 & 0.380 & 0.509494 \\
3 & 0.00067 & 0.443 & 0.759494 \\
$2+4$ & 0.00046 & 0.497 & \\
3 & 0.00033 & 0.516 & \\
$2+4$ & 0.00025 & 0.585 & \\
$1+5$ & 0.00023 & 0.589 & \\
3 & 0.00016 & 0.658 &
\end{tabular}

Table 1 Successive iterations of our minimization scheme with gradual adjustment of the local multiplet basis at half band-filling for $U=2, J / U=0.2, \zeta=0.2$ (SOC), $\Delta=$ 0.2 (CFS); $n_{\text {active: }}$ particle number of the subspaces where non-diagonal variational parameters are included; $\Delta E_{\text {tot }}$ : error in total energy; $t_{\mathrm{CPU}}$ : CPU time for the present, the CON1, and a full Gutzwiller minimization.

In table 1 we present the error $\Delta E_{\text {tot }}$ after each step of the iteration, and the corresponding required CPU time $t_{\mathrm{CPU}}$, relative to the CPU time $t_{\mathrm{CPU}}^{\mathrm{full}}$ for a full Gutzwiller minimization. For comparison, we also show the CPU time $t_{\mathrm{CPU}}^{\mathrm{CON} 1}$ of some CON1 minimizations that lead to the same accuracy, cf. the red dashed line in Fig. 8

Note that the not strictly-monotonic behavior of $t_{\mathrm{CPU}}^{\mathrm{CON} 1}$ in table 1 is genuine. While the CPU time for the 'BroydenFletcher-Goldfarb-Shanno' (BFGS) minimization' with respect to $\tilde{\lambda}$ generally increases with the total number $N_{\mathrm{v}}$ of variational parameters, the constraint enforcing 'Penalty and Augmented Lagrangian method' (PALM) contains a 
certain degree of arbitrariness, see Ref. [22]. Therefore, it can occasionally happen that, when increasing $N_{\mathrm{v}}$, the PALM scheme needs less loops. This can compensate the increase of $T_{\mathrm{CPU}}^{\mathrm{CON} 1}$ from the BFGS minimization.

Table 1 shows that the convergence of our algorithm is rather fast, especially in the first few steps where the energy makes large gains with modest numerical effort. Only when we try to obtain very, and probably unnecessarily, accurate results, it will eventually be outperformed by a CON1 minimization.

Our algorithm overcomes the computer memory barrier of the CON1 method because its accuracy depends on the allocated CPU time. Most importantly, the iterative algorithm can be expected to converge for any multi-orbital system. Therefore, we think that it is the most promising candidate to be used for a general Gutzwiller minimization code that is needed in a Gutzwiller-DFT program package [27,28, 29].

5 Summary In this work, we calculated the Gutzwiller variational ground-state energy for a three-orbital Hubbard model in three dimensions within the Gutzwiller approximation. For our three-orbital model it is possible to carry out a minimization with respect to the most general Gutzwiller Ansatz that includes all elements in the Hermitian variational-parameter matrix and thus serves as a benchmark test for approximations.

As observed in earlier studies, a variational Ansatz with a diagonal variational-parameter matrix is very accurate in systems with a high point-group symmetry. However, when the symmetry is lower, e.g., by an inclusion of the spin-orbit coupling, non-diagonal variational parameters become very important, e.g., for the quasi-particle band structure.

An obvious strategy to improve a purely diagonal Ansatz consists in a brute-force inclusion of the most important non-diagonal variational parameters. However, in cases with a low symmetry, this approach can be inefficient and ineffective, and may lead to significant errors in systems with more than three orbitals. Therefore, we propose a more suitable algorithm that is based on a gradual adaption of the local multiplet basis. This method converges quite rapidly, especially in its first few iteration steps. Moreover, since it is only limited by the available CPU time and not by memory constraints, we consider it the most promising candidate to be used in a general Gutzwiller minimization program.

Acknowledgements This work was supported in part by the Priority Programme 1458 of the Deutsche Forschungsgemeinschaft (DFG) under GE 746/10-1. We thank R. Schade for valuable discussions on optimization algorithms. The authors gratefully acknowledge the computing time granted by the John-vonNeumann Institute for Computing (NIC), and provided on the supercomputer JURECA at Jülich Supercomputing Centre (JSC) under project no. HDO08.

\section{References}

[1] G. R. Stewart, Rev. Mod. Phys. 83, 1589 (2011).

[2] P. Dai, Rev. Mod. Phys. 87, 855 (2015).

[3] O. Andersen and L. Boeri, Annalen der Physik 523, 8 (2011).

[4] M. Gutzwiller, Phys. Rev. Lett. 10, 159 (1963).

[5] M. Gutzwiller, Phys. Rev. 134, A923 (1964).

[6] W. Metzner and D. Vollhardt, Phys. Rev. Lett. 59, 121 (1987).

[7] F. Gebhard, Phys. Rev. B 41, 9452 (1990).

[8] T. Schickling, F. Gebhard, and J. Bünemann, Phys. Rev. Lett. 106, 146402 (2011).

[9] T. Schickling, F. Gebhard, J. Bünemann, L. Boeri, O. K. Andersen, and W. Weber, Phys. Rev. Lett. 108, 036406 (2012).

[10] J. Bünemann, T. Schickling, and F. Gebhard, Europhys. Lett. 98, 27006 (2012).

[11] J. Kaczmarczyk, J. Spałek, T. Schickling, and J. Bünemann, Phys. Rev. B 88, 115127 (2013).

[12] J. Kaczmarczyk, Phil. Mag. 95, 563 (2015).

[13] J. Kaczmarczyk, T. Schickling, and J. Bünemann, physica status solidi (b) 252, 2059 (2015).

[14] M. M. Wysokiński, J. Kaczmarczyk, and J. Spałek, Phys. Rev. B 92, 125135 (2015).

[15] M. M. Wysokiński, J. Kaczmarczyk, and J. Spałek, arxiv: 1510.00224.

[16] J. Kaczmarczyk, J. Bünemann, and J. Spałek, New Journal of Physics 16, 073018 (2014).

[17] K. zu Münster and J. Bünemann, (unpublished).

[18] J. C. Slater and G. F. Koster, Phys. Rev. 94, 1498 (1954).

[19] J. Bünemann, W. Weber, and F. Gebhard, Phys. Rev. B 57, 6896 (1998)

[20] J. Bünemann, F. Gebhard, and W. Weber, in: Frontiers in Magnetic Materials, edited by A. Narlikar, (Springer, Berlin, 2005).

[21] J. Bünemann, F. Gebhard, T. Schickling, and W. Weber, physica status solidi (b) 249, 1282 (2012).

[22] J. Bünemann, T. Linneweber, U. Löw, F. Anders, and F. Gebhard, arXiv:1603.07544 (2016).

[23] J. Nocedal and S. J. Wright, Numerical Optimization, 2nd edition (Springer, New York, 2006).

[24] J. Bünemann, F. Gebhard, and R. Thul, Phys. Rev. B 67, 75103 (2003).

[25] G. Borghi, M. Fabrizio, and E. Tosatti, Phys. Rev. B 90, 125102 (2014).

[26] L. Du, L. Huang, and X. Dai, Eur. Phys. J. B 86, 94 (2013).

[27] K. M. Ho, J. Schmalian, and C. Z. Wang, Phys. Rev. B 77, 073101 (2008).

[28] X. Deng, X. Dai, and Z. Fang, Europhys. Lett. 83, 37008 (2008).

[29] T. Schickling, J. Bünemann, F. Gebhard, and W. Weber, New Journal of Physics 16, 93034 (2014). 